\title{
Improving the Strategy and Performance of Molecularly Imprinted Polymers using Crosslinking Functional
}

\section{Monomers}

Martha Sibrian-Vazquez, David A. Spivak*

Department of Chemistry, Louisiana State University, 70803.

Phone: (225) 578 2868, Fax: (225) 578 3458, E-mail: David_Spivak@chem.lsu.edu

\section{Supporting Information}

$\begin{array}{lc} & \text { Page } \\ \text { General Experimental } & \mathrm{S} 3 \\ { }^{1} \mathrm{H} \text { NMR compound } 8 & \mathrm{~S} 4 \\ { }^{13} \mathrm{C} \text { NMR compound } 8 & \mathrm{~S} 5 \\ { }^{1} \mathrm{H} \text { NMR compound } 3 & \mathrm{~S} 6 \\ { }^{13} \mathrm{C} \text { NMR compound } 3 & \mathrm{~S} 7 \\ { }^{1} \mathrm{H} \text { NMR compound } 10 & \mathrm{~S} 8 \\ { }^{13} \mathrm{C} \text { NMR compound } 10 & \mathrm{~S} 9 \\ { }^{1} \mathrm{H} \text { NMR compound } 11 & \mathrm{~S} 10 \\ { }^{13} \mathrm{C} \text { NMR compound } 11 & \mathrm{~S} 11 \\ { }^{1} \mathrm{H} \text { NMR compound } 12 & \mathrm{~S} 12 \\ { }^{13} \mathrm{C} \text { NMR compound } 12 & \mathrm{~S} 13\end{array}$


${ }^{1} \mathrm{H}$ NMR compound $13 \quad$ S14

${ }^{13}$ C NMR compound $13 \quad \mathrm{~S} 15$

${ }^{1} \mathrm{H}$ NMR compound $2 \quad$ S16

$\begin{array}{ll}{ }^{13} \mathrm{C} \text { NMR compound } 2 & \text { S17 }\end{array}$

${ }^{1} \mathrm{H}$ NMR compound $5 \quad$ S18

${ }^{13}$ C NMR compound 5 $\quad$ S19

$\begin{array}{ll}{ }^{1} \mathrm{H} \text { NMR compound } 16 & \text { S20 }\end{array}$

${ }^{13} \mathrm{C}$ NMR compound $16 \quad$ S21

${ }^{1}$ H NMR compound $6 \quad$ S22

${ }^{13} \mathrm{C}$ NMR compound $6 \quad$ S23 


\section{Experimental}

\section{General}

Unless otherwise indicated, chemicals were commercially available and used without further purification. Solvents were obtained from commercial suppliers and used as is. Reactions were monitored by thin-layer chromatography, fractions being visualized by UV light or iodine. Column chromatography was carried out with flash silica gel. All reported yields were determined after purification. Chemical shifts $(\delta)$ are given in ppm relative to $\mathrm{CDCl}_{3}\left(7.27 \mathrm{ppm},{ }^{1} \mathrm{H} ; 77.0 \mathrm{ppm},{ }^{13} \mathrm{C}\right)$ unless otherwise indicated. IR spectra were obtained as neat samples unless otherwise indicated. High-resolution mass spectra (HRMS) were obtained under fast atom bombardent (FAB, liquid sims) ionization or electrospray ionization (EI). Imprinted polymerization was performed in a photochemical turntable reactor, which was immersed in a constant temperature bath. A standard laboratory UV light source jacketed in a borosilicate double-walled immersion well was placed at the center of the turntable reactor. HPLC columns were packed using a solvent delivery module, into stainless steel columns (length, $7.5 \mathrm{~cm}$, i.d. $2.1 \mathrm{~mm}$ ) to full volume for chromatographic experiments. HPLC analyses were performed isocratically at room temperature $\left(21^{\circ} \mathrm{C}\right)$ with $\mathrm{UV}$ detector. 

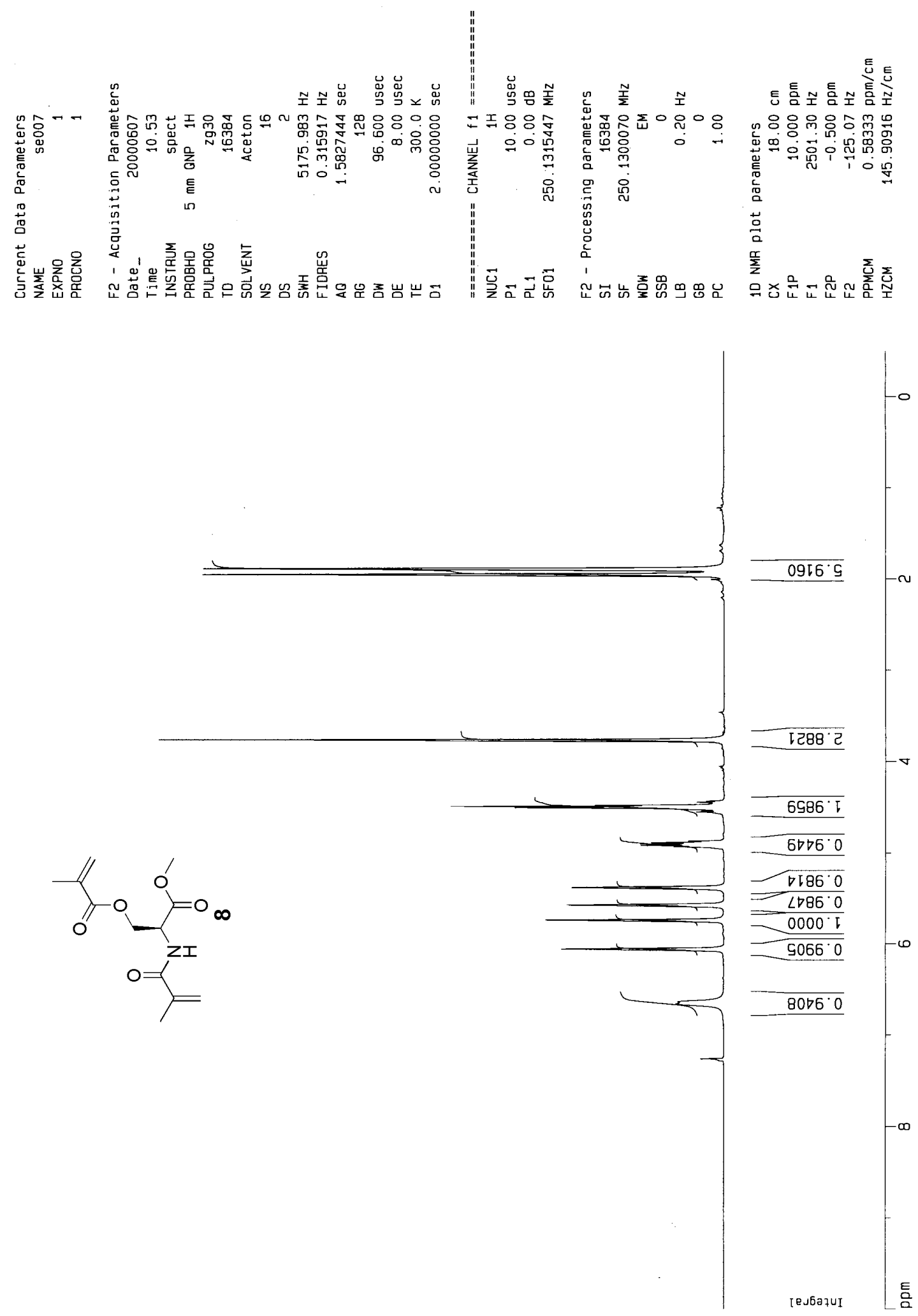

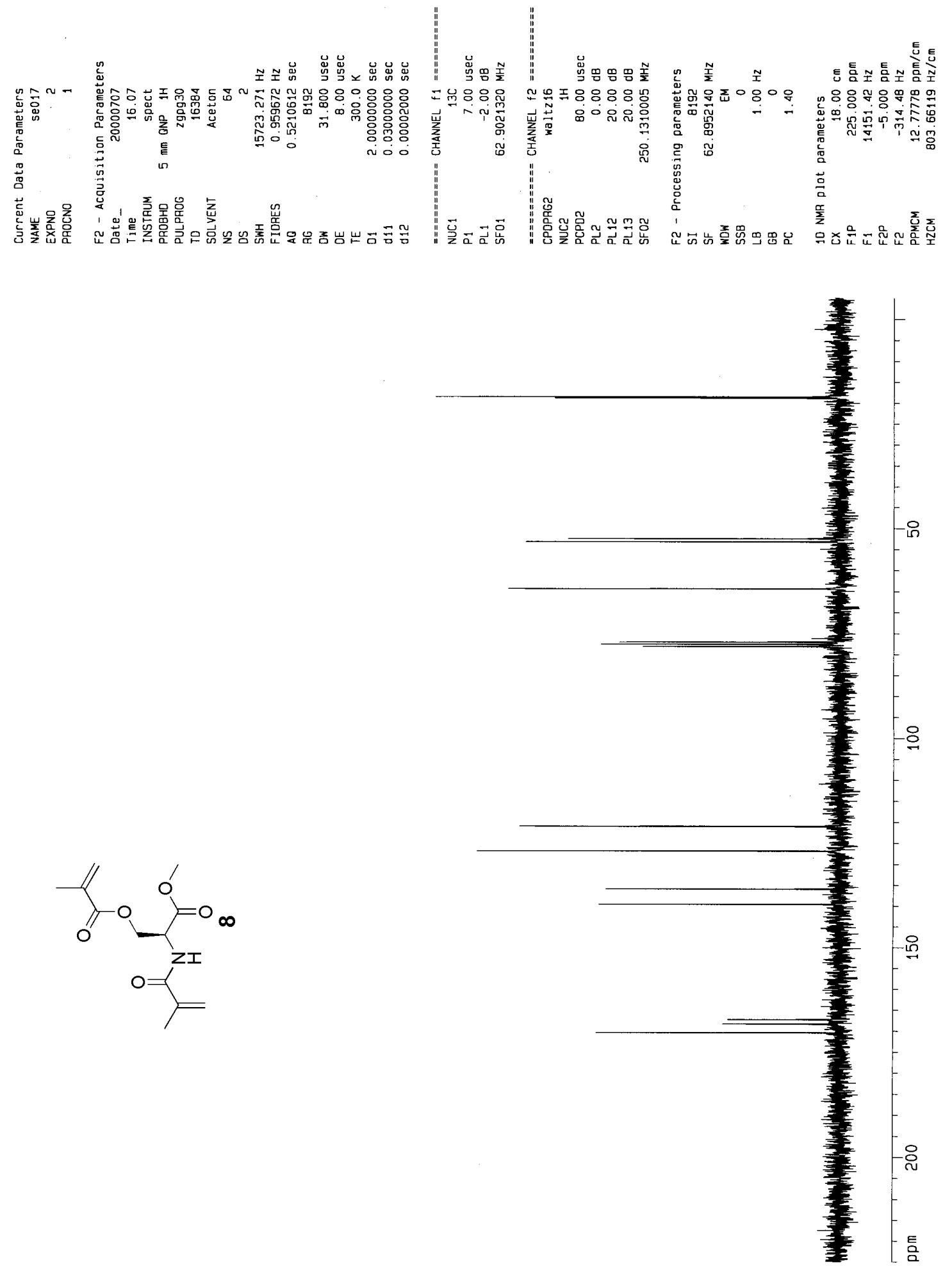

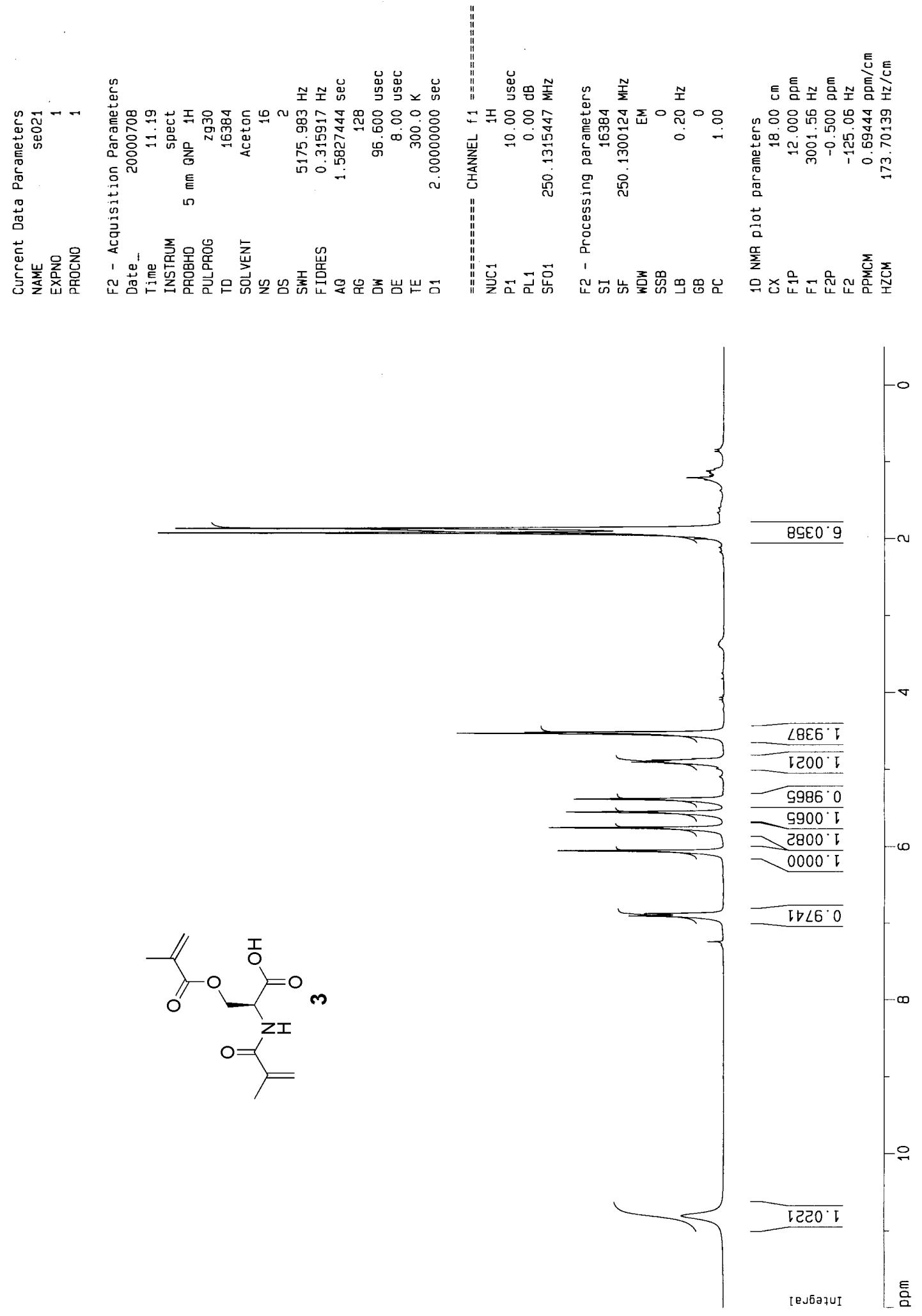

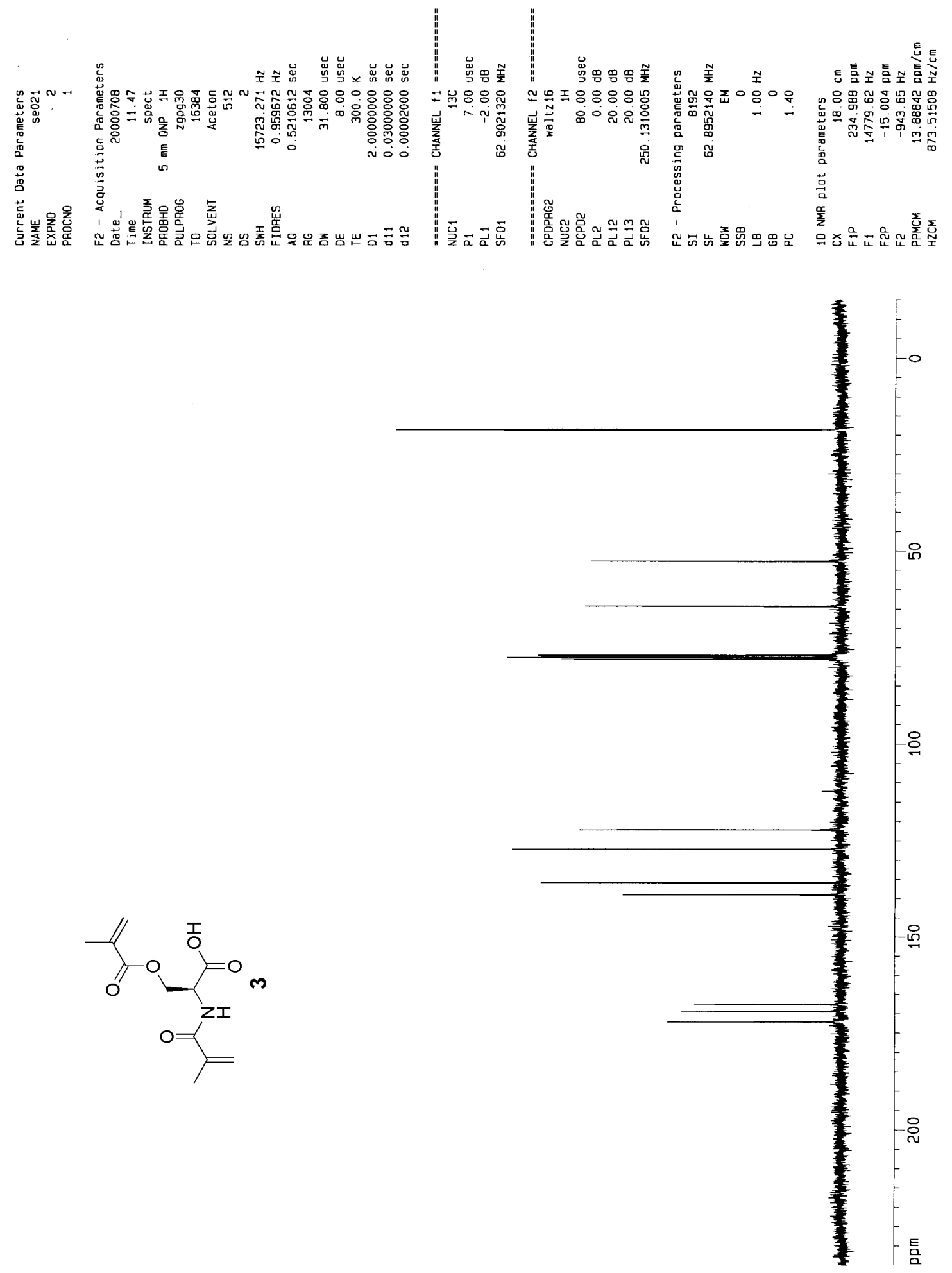

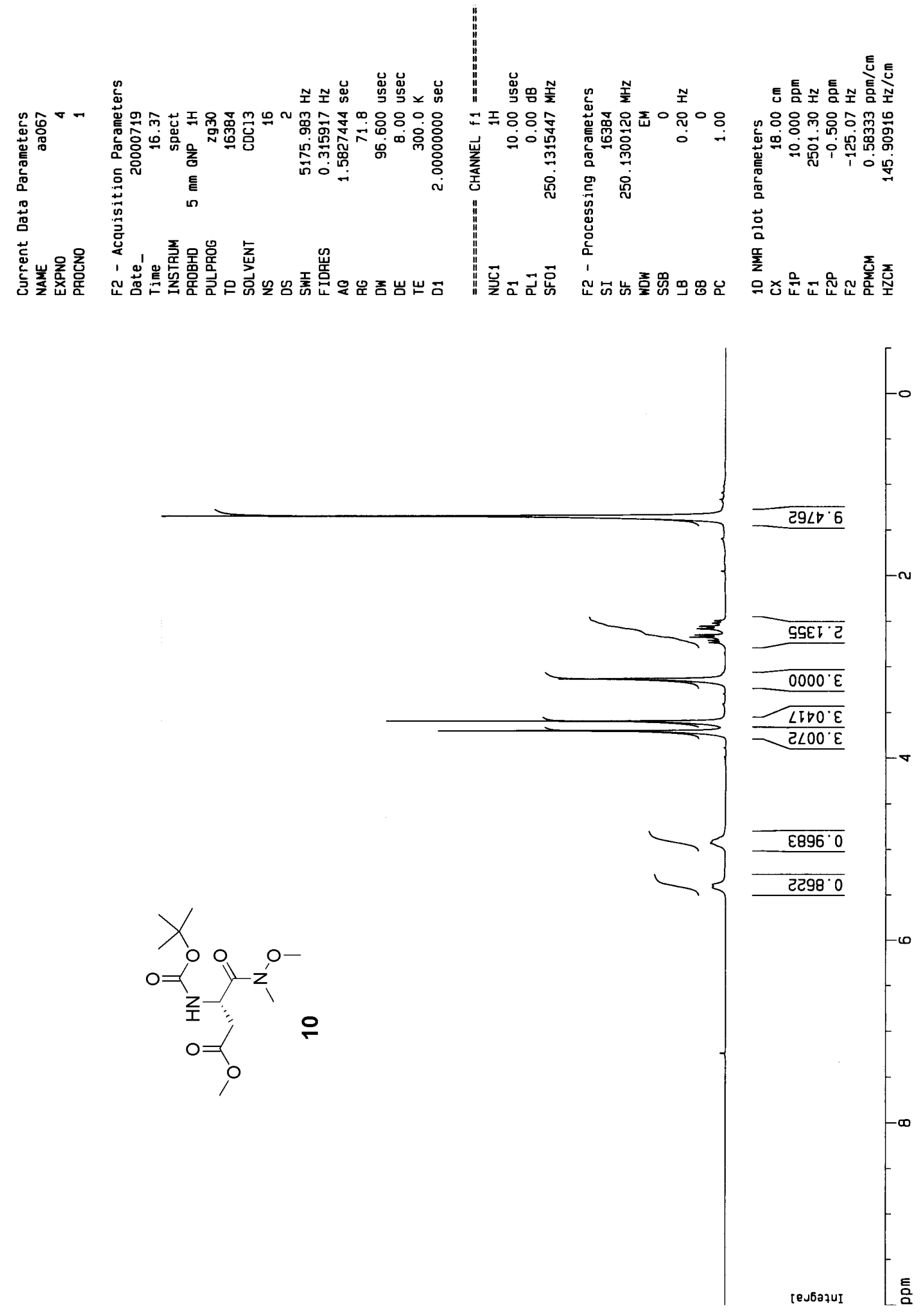

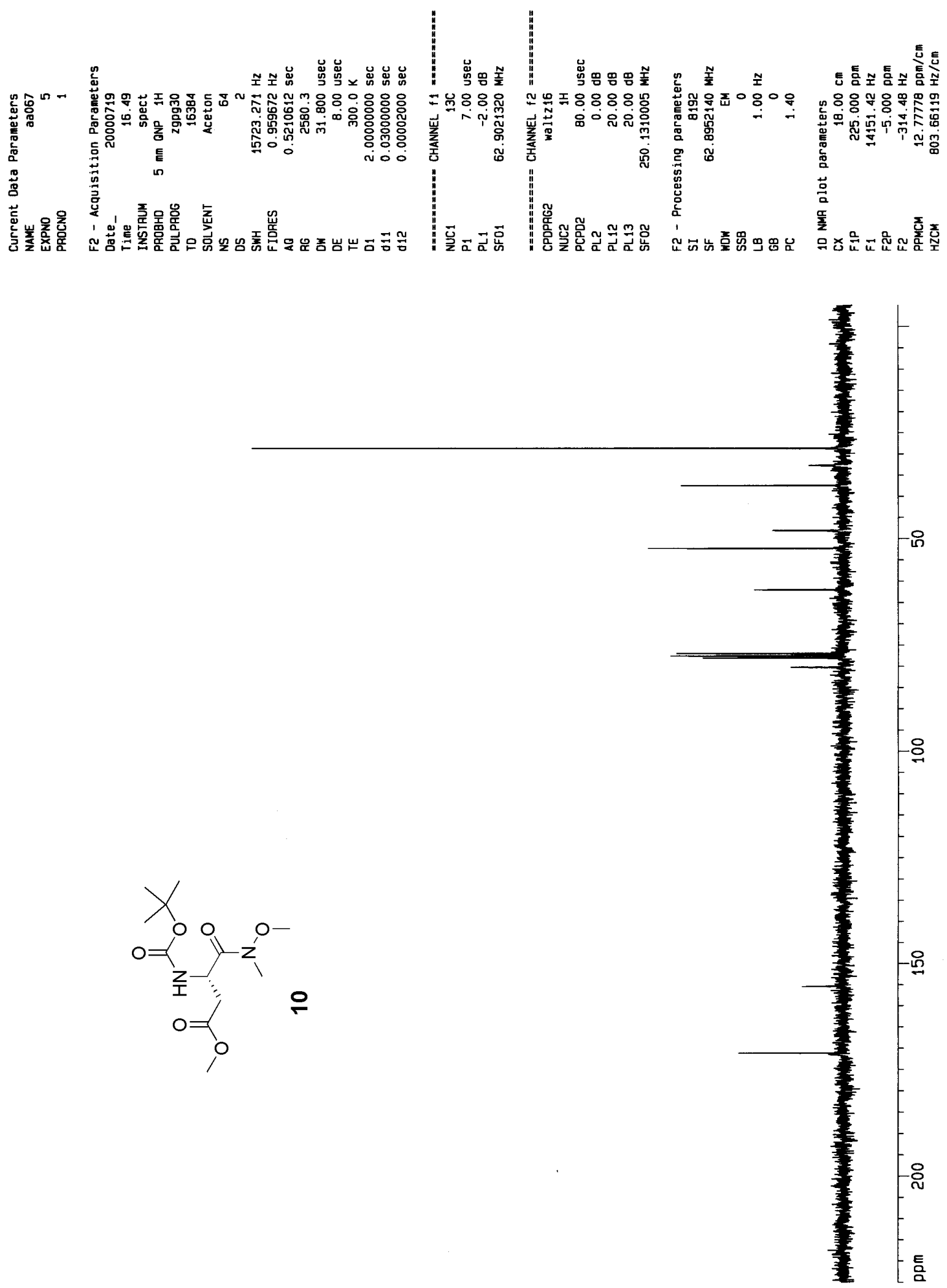

S9 

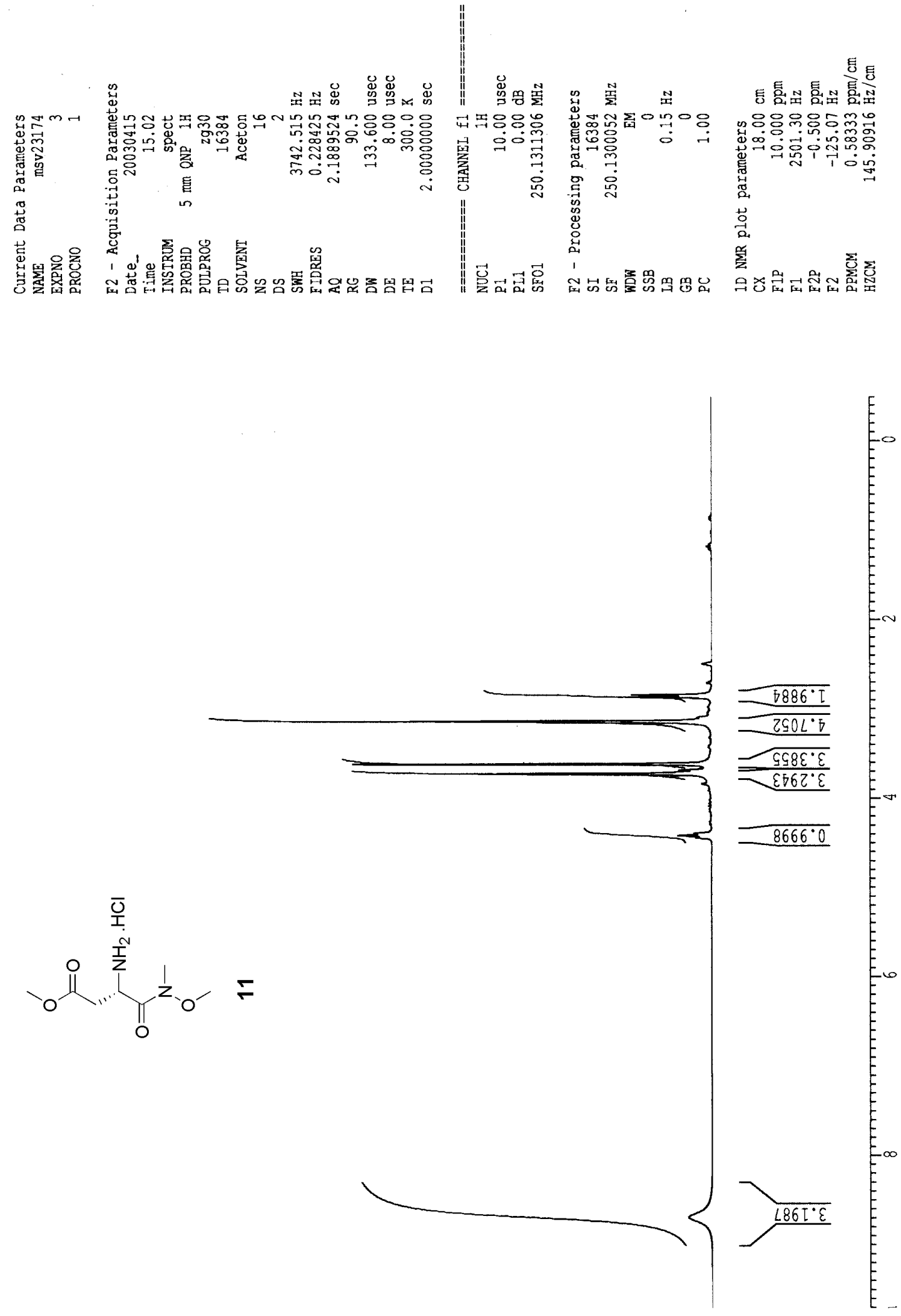

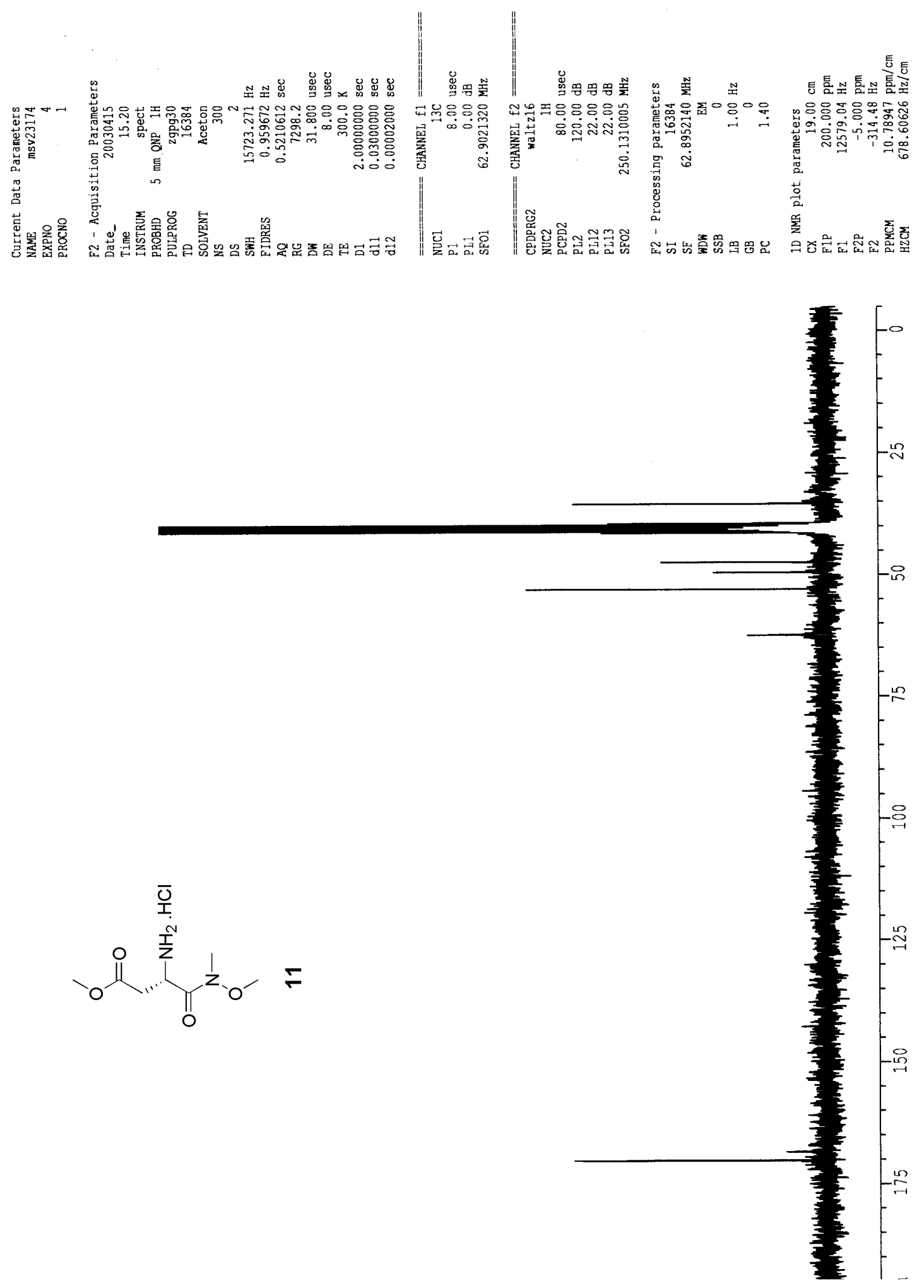

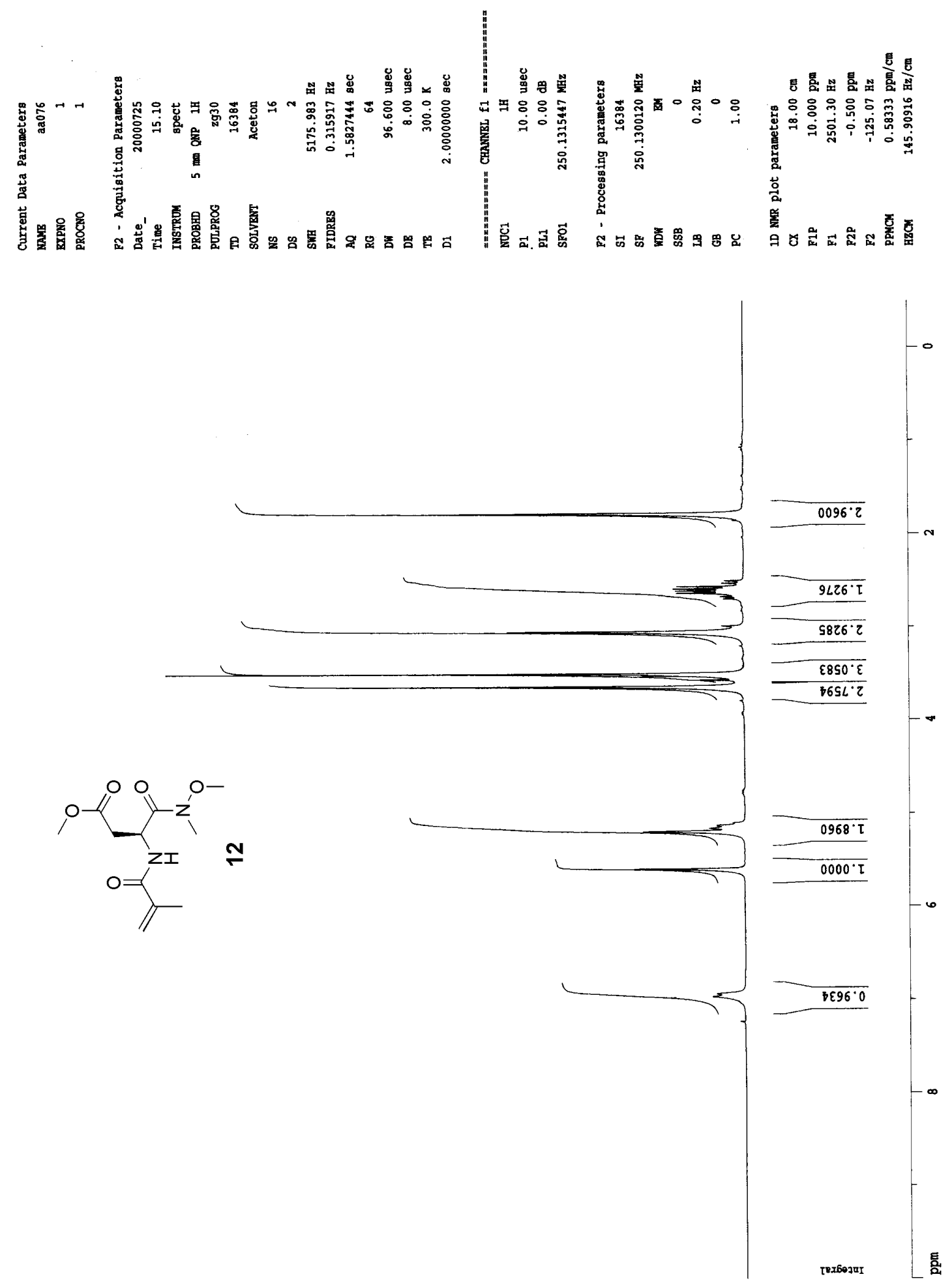

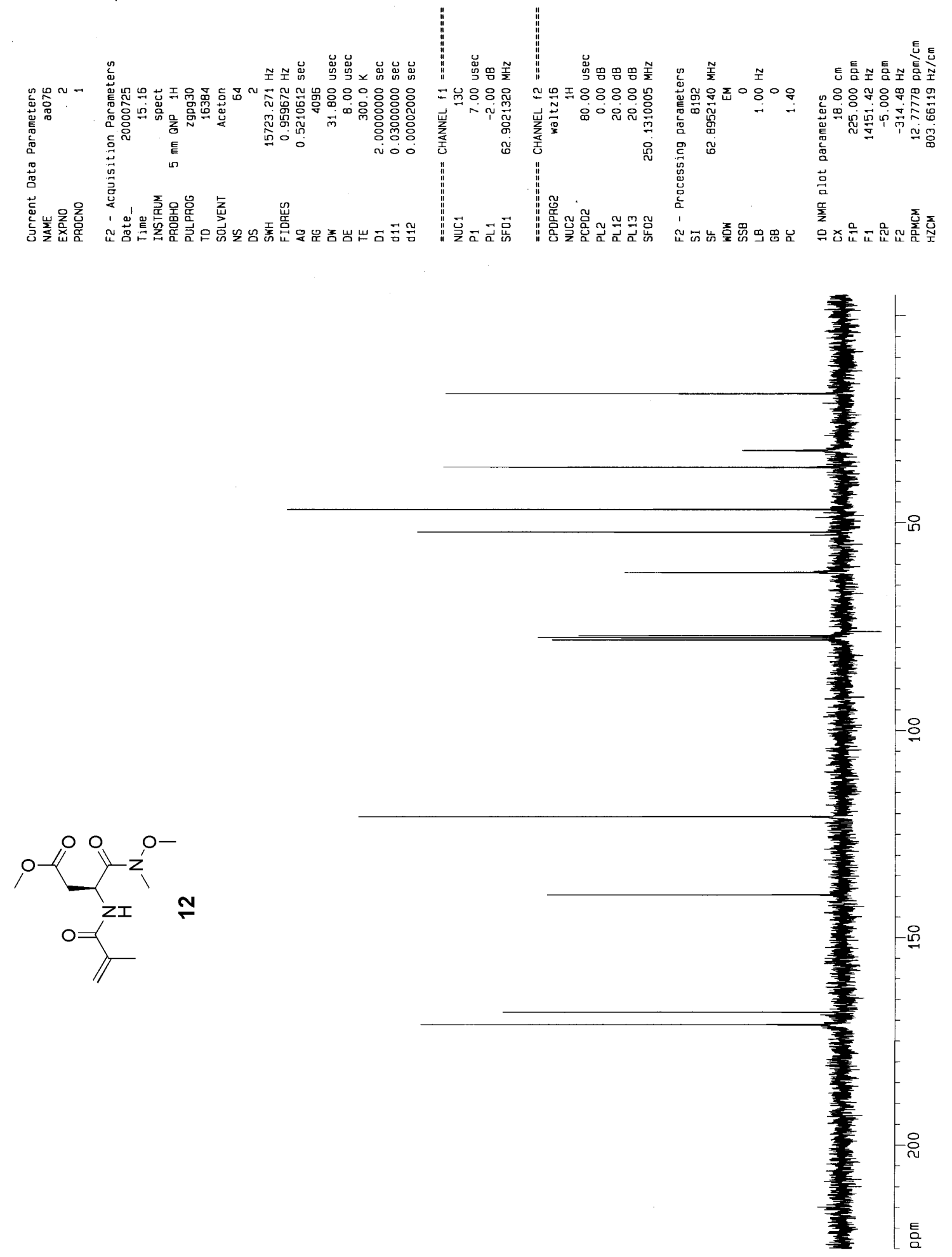

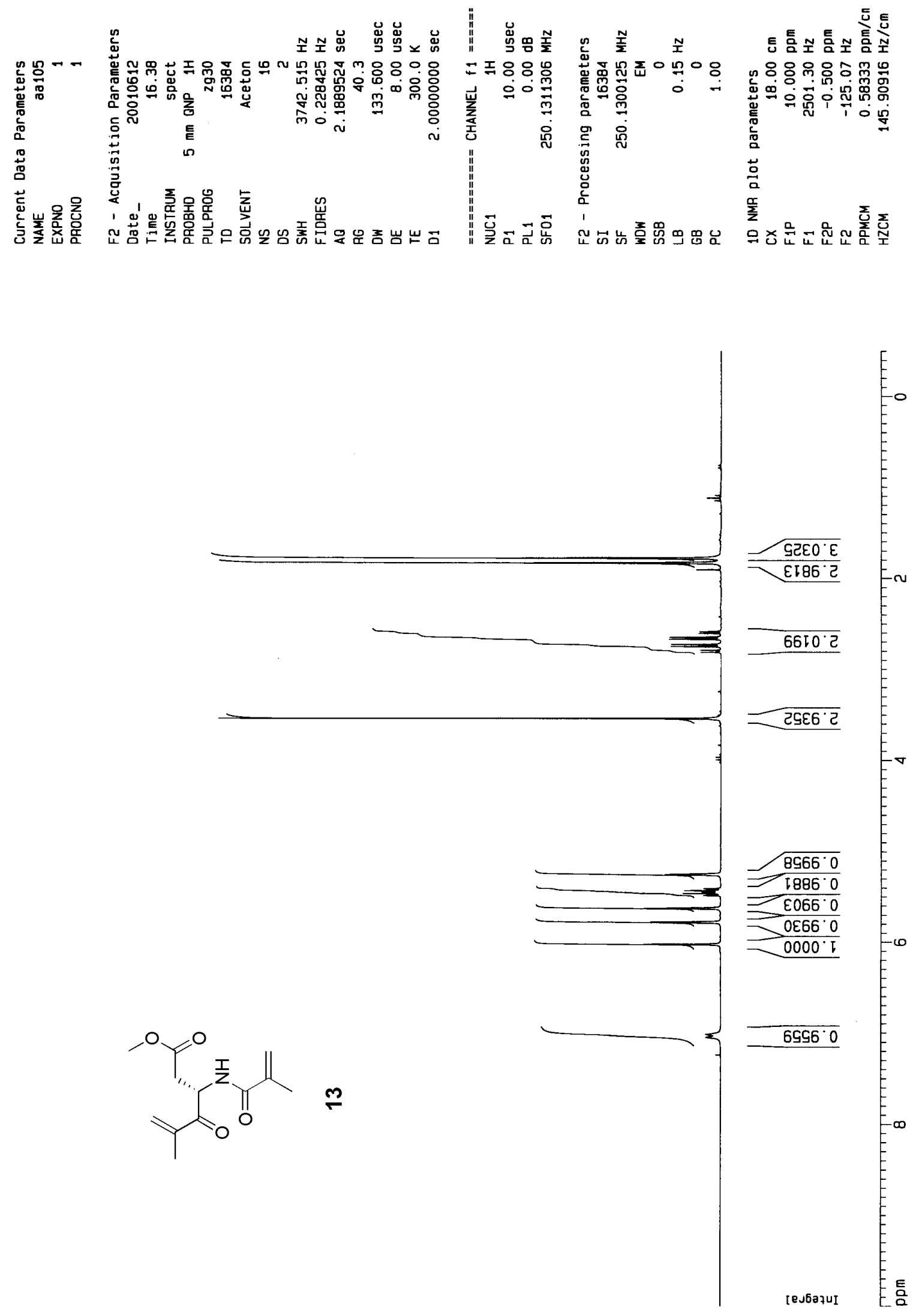

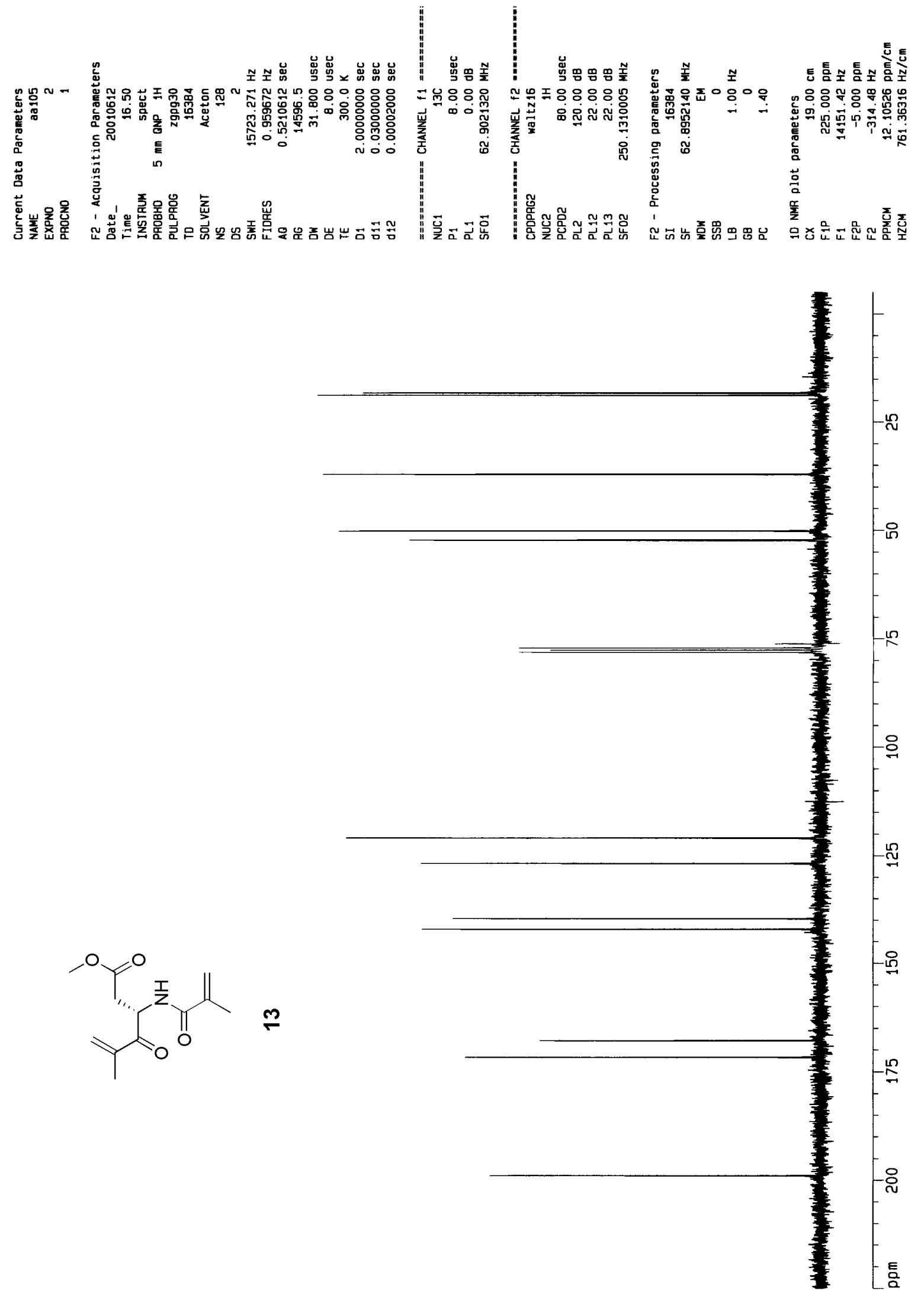

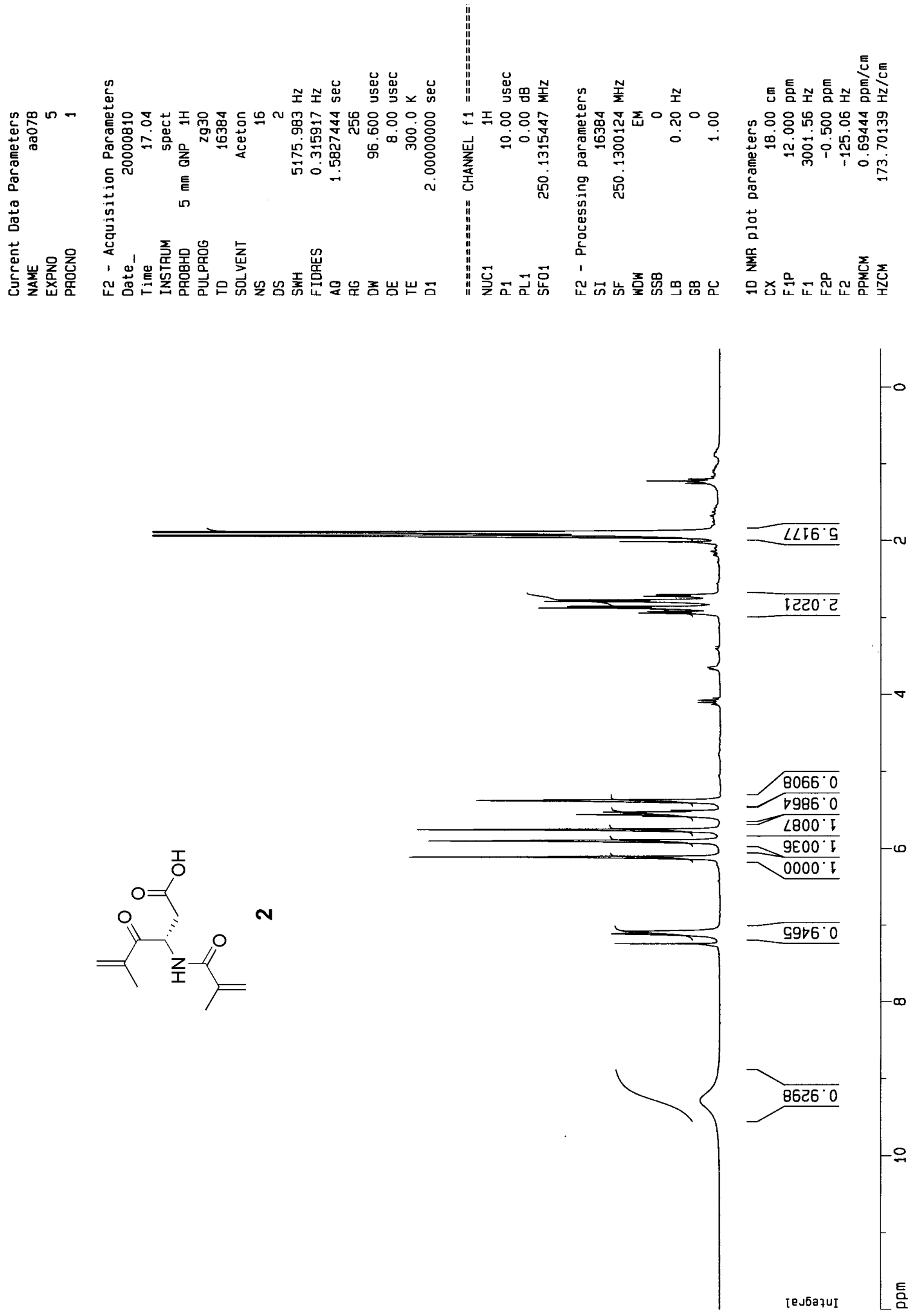


$$
1
$$



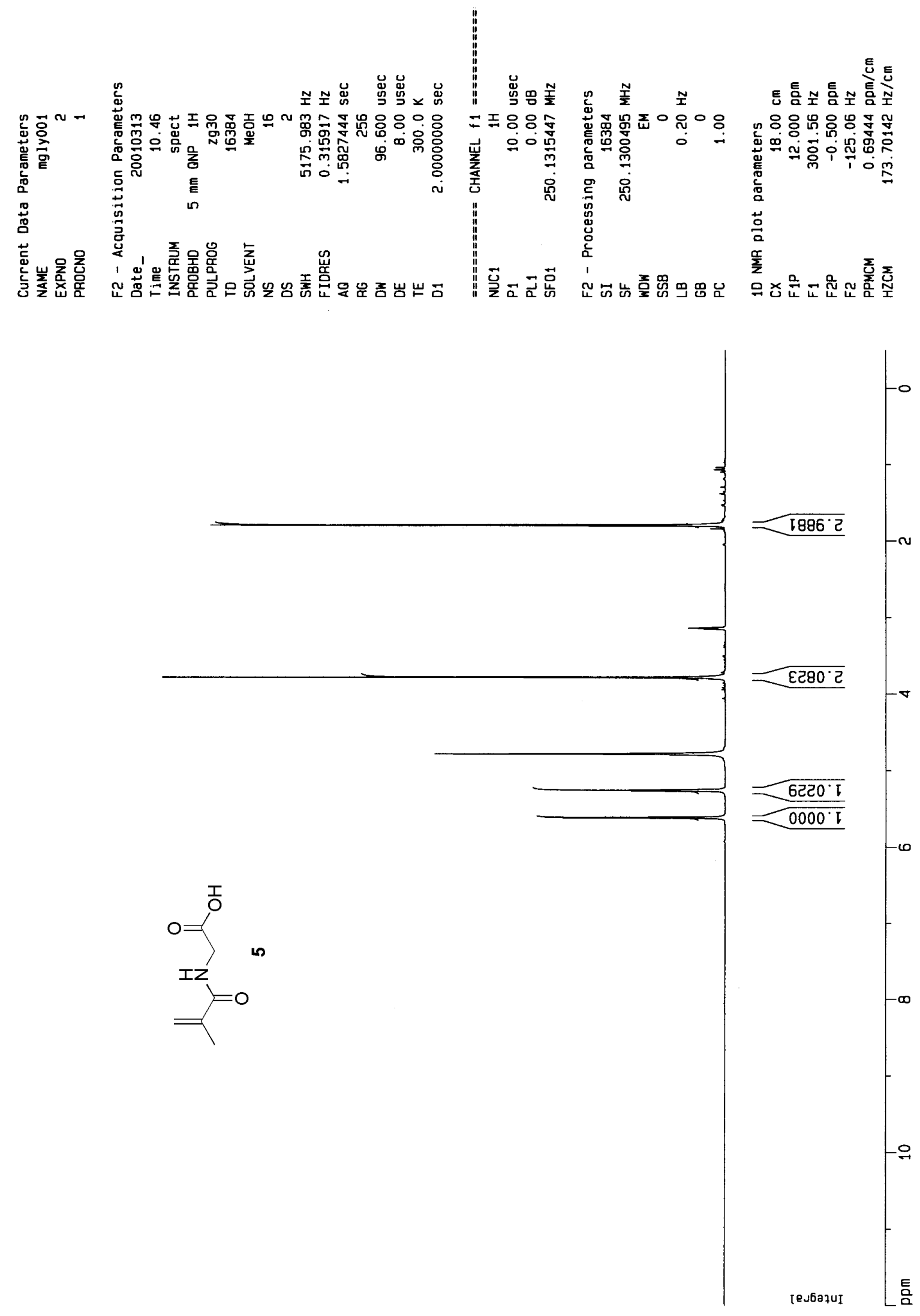


$$
7
$$



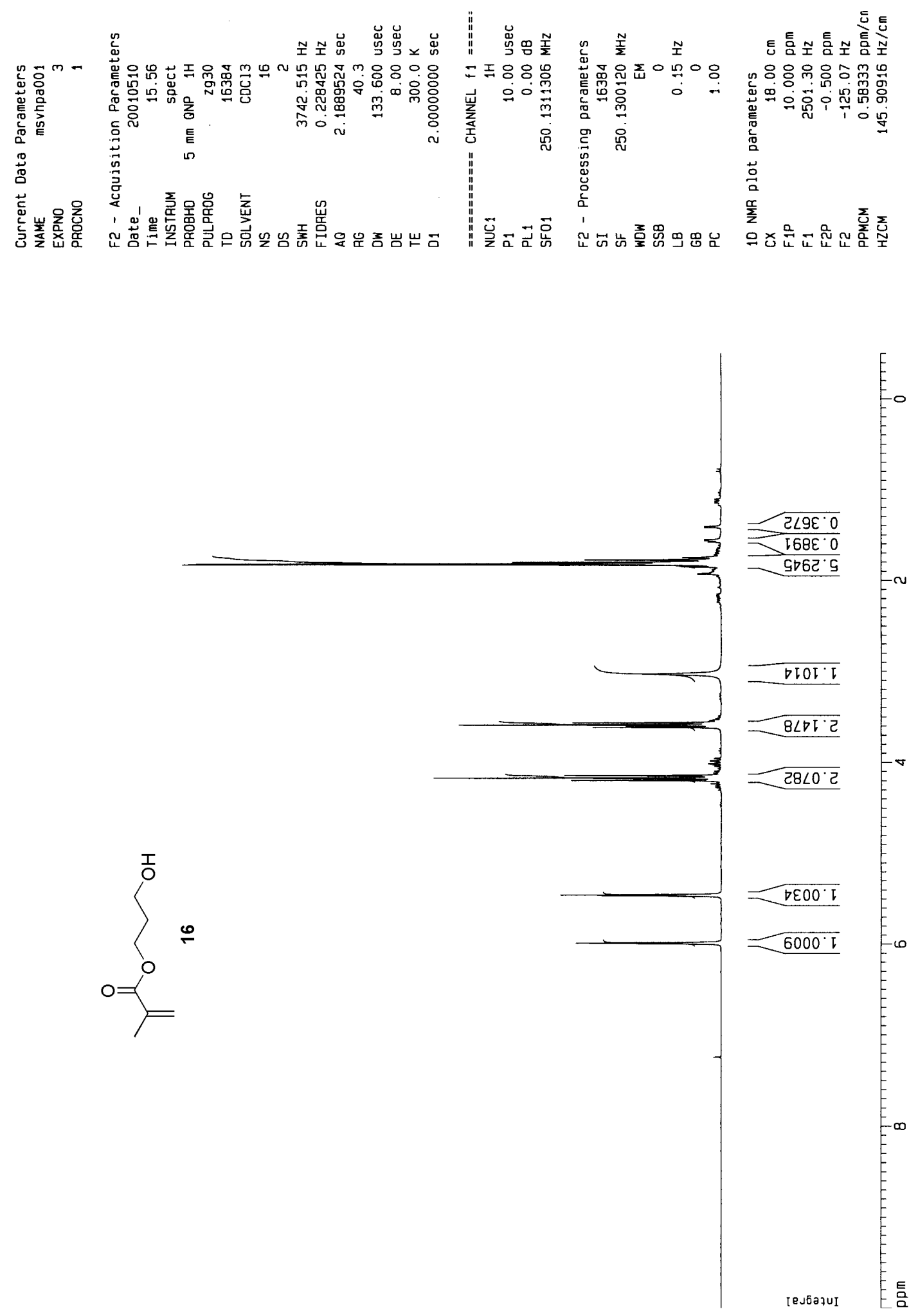

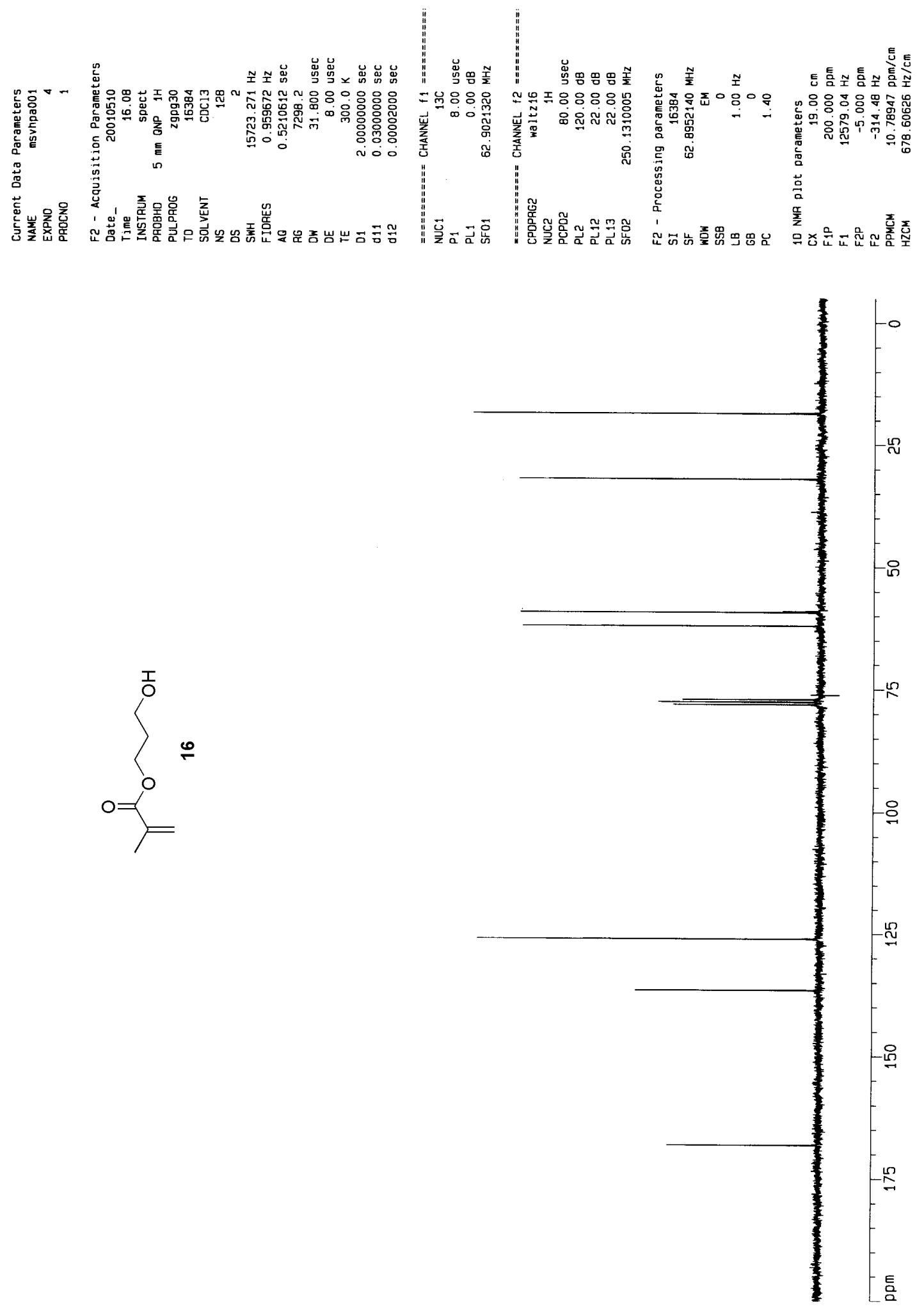

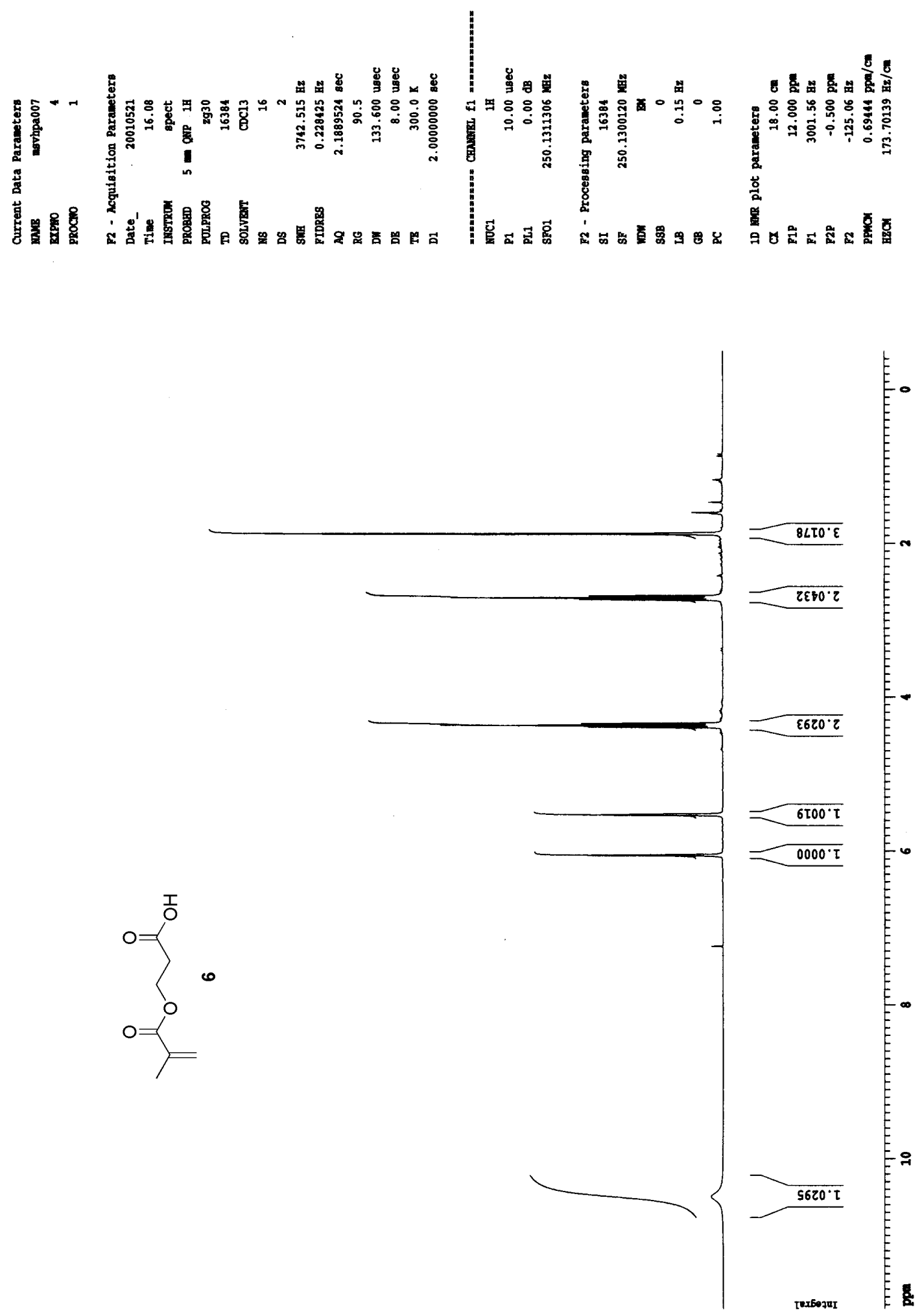

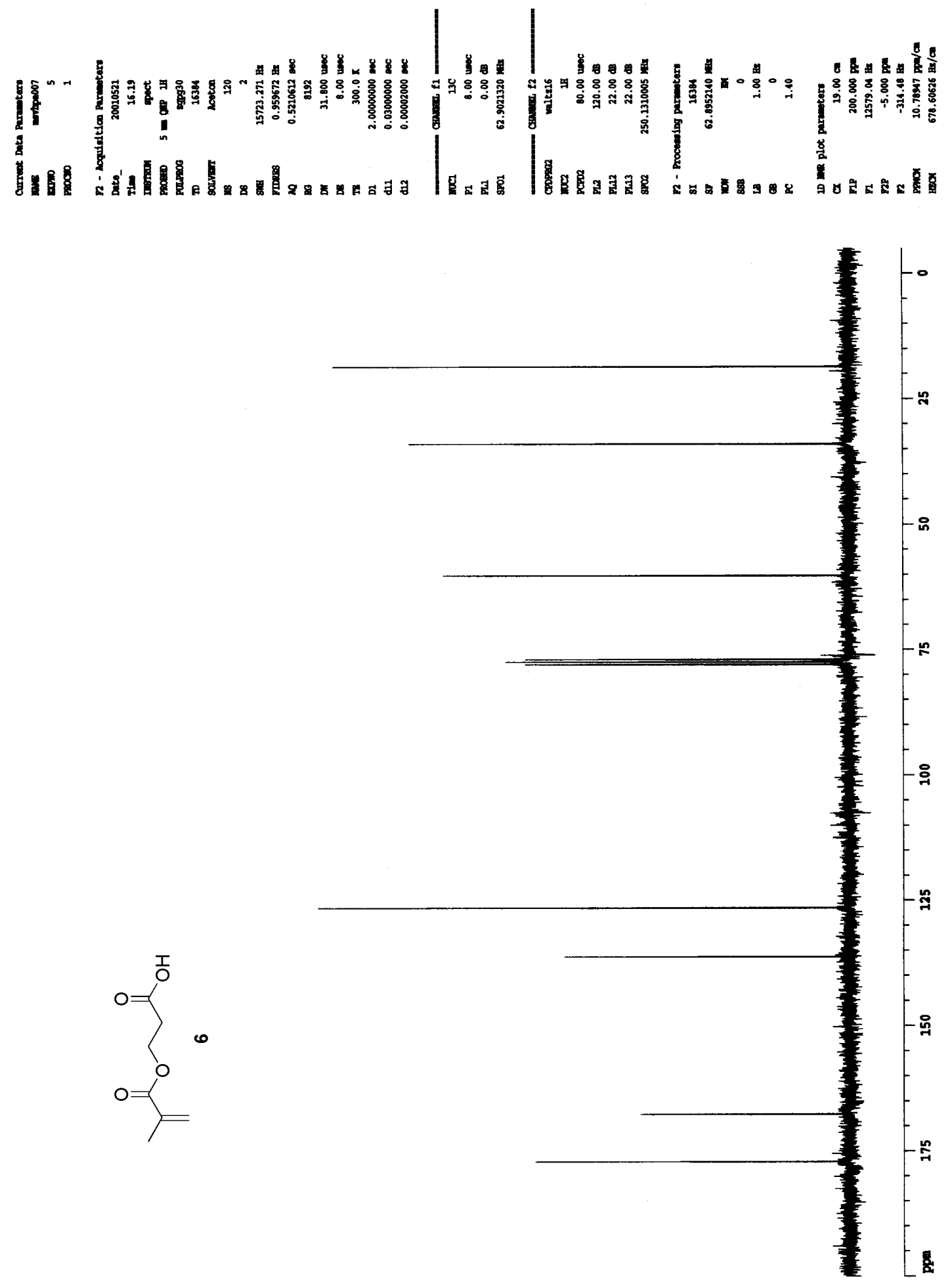